\title{
Diseño curricular de ingeniería industrial según la tendencia de internacionalización
}

\author{
CURRICULAR DESIGN OF INDUSTRIAL ENGINEERING ACCORDING TO THE \\ INTERNATIONALIZATION TREND
}

\section{Resumen}

Palabras clave: Diseño curricular; ingeniería industrial; internacionalización; educación a distancia
La presente investigación tuvo como propósito analizar el diseño curricular actual de la carrera Ingeniería Industrial de la Universidad Nacional Abierta de Venezuela (UNA), en términos de las tendencias curriculares internacionalización para la educación universitaria. El evento de estudio estuvo representado por el contenido del diseño curricular de la carrera Ingeniería Industrial de la UNA. La técnica para la recolección de información fue la revisión documental y el instrumento una matriz de análisis, la cual se aplicó para el criterio de análisis internacionalización del currículo. Para el procesamiento de la información se utilizó el análisis por composición. Se concluye que el diseño curricular actual de la carrera Ingeniería Industrial de la Universidad Nacional Abierta de la República Bolivariana de Venezuela, adolece de elementos que destacan en la tendencia de internacionalización del currículo en la educación universitaria.

The present research was analytical and had the general objective of analyzing the current curricular design of the Industrial Engineering degree at the National Open University of Venezuela (UNA), in terms of internationalization trends of the curriculum for university education. The study event was represented by the content of the curriculum design of the Industrial Engineering degree at UNA. The technique for collecting information was the documentary review and the instrument an analysis matrix, which was applied to the internationalization analysis criteria of the curriculum. For the information processing the analysis by composition was used. It is concluded that the current curricular design of the Industrial Engineering degree at the National Open University of the Bolivarian Republic of Venezuela suffers from elements that stand out in the internationalization trend of the curriculum in university education.

\section{Keywords:}

Curriculum design; industrial engineering; internationalization; distance education 
INTRODUCCIÓN

La tendencia de la
internacionalización del currículo cobra auge por la modernización de los medios de información y comunicación, la cual se basa en los desarrollos científicostecnológicos de las últimas décadas. Estos avances han permitido el acortamiento de las distancias entre los países, superando las fronteras que limitaban el intercambio de conocimiento, culturas, entre otros.

La referida tendencia ha impactado a nivel mundial sobre el diseño curricular de las carreras que se imparten en las universidades, pues muchas universidades realizan alianzas estratégicas para el desarrollo de las funciones de docencia, investigación y extensión universitaria.

En ese sentido, es a través de los diseños curriculares, acompañados por lineamientos políticos institucionales, así como de un basamento tecnológico de avanzada, que las universidades pueden trascender más allá de sus fronteras. De tal manera que se pueda apreciar la internacionalización del currículo, en pro del desarrollo del talento humano que hace vida en las universidades y se expande en el ámbito local, nacional y global para difundir su conocimiento.

Las universidades, como instituciones que contribuyen al desarrollo de las naciones, dadas sus funciones de docencia, investigación y extensión, son las responsables de la formación profesional del talento humano requerido por los países, por lo que están en conexión directa con las necesidades prácticas de la sociedad en el sentido de impulsar los procesos de desarrollo en empresas de producción y servicios, tanto privadas como públicas (Altbach, 2008).

Así, los profesionales egresados de las instituciones universitarias deben contar con los conocimientos, habilidades y destrezas -adquiridas en su trayecto académico-, para enfrentarse a los cambios exigidos por la sociedad contemporánea. Una sociedad altamente tecnificada, donde la información requerida para la toma de decisiones se obtiene en tiempo real desde cualquier parte del mundo. De igual manera, estos cambios generados deben impulsar el análisis de los procesos educativos de las universidades, esto con el fin de estar en sintonía con las exigencias sociales, económicas, tecnológicas y culturales del mundo actual.

A esta realidad no escapan las universidades venezolanas, las cuales requieren rediseñar su práctica, trascendiendo la visión esencialmente local y nacional de la educación tradicional, asumiendo una perspectiva y conciencia global. Transformándose en instituciones donde se formen ciudadanos con perspectivas globales, capaces de transformar la sociedad, impulsar la democracia, la comprensión y el respeto a las diferentes culturas, así como de promover la conservación del medio ambiente.

En consecuencia, siguiendo a Henao y Samoilovich (2010) se hace necesario el desarrollo de un diseño curricular que transmita a los futuros profesionales las "actitudes, competencias y conocimientos que les permitan desenvolverse y trabajar en un mundo globalizado, interconectado 
y multicultural" (s.n.). Toda vez que el diseño curricular es uno de los componentes fundamentales del proceso educativo.

El diseño curricular se caracteriza por su dinamismo y flexibilidad lo que le permite adaptarse a los cambios sociales, económicos, políticos, tecnológicos, entre otro, a los cuales ha estado sometido a lo largo del tiempo. No obstante, estos cambios deben ser racionales y sujetos a una constante revisión para su adecuación. Obviamente, que estos cambios propician en las instituciones de educación superior procesos de reflexión sobre lo que se está haciendo, su propósito y reorientación de su concepción curricular, esto en consideración de que las universidades son entes de transformación social, de generación y difusión de conocimientos.

Es importante destacar que los diseños curriculares de las carreras universitarias no solo deben responder al contexto inmediato, sino además enmarcar una visión global, nacional, regional, integral y prospectiva, lo que exige repensarlos con criterios de rigurosidad, calidad, creatividad, modernidad; en aras de proponer configuraciones educativas $y$ organizacionales, pertinentes y con capacidad de plantearse escenarios futuros, direccionados hacia la interrelación, interconexión, transformación y por ende, comprometidos con el destino social de la humanidad en sentido amplio.

En ese sentido, es a través de los diseños curriculares innovadores, acompañados de un basamento tecnológico de avanzada, que las universidades pueden trascender más allá de sus fronteras. De tal manera que se pueda apreciar la internacionalización del currículo, en pro del desarrollo del talento humano que hace vida en las universidades y se expande para difuminar su conocimiento en el ámbito local, nacional y global.

De allí, el reconocimiento que tienen las tecnologías de la información y comunicación en el ámbito de las universidades. Más aún en aquellas que basan su modelo educativo en la educación abierta y a distancia como la Universidad Nacional Abierta (UNA), la cual constituye la única institución universitaria venezolana fundada bajo los principios de la educación abierta y a distancia, modalidad de estudio que potencia el intercambio de saberes y conocimiento, más allá de las fronteras nacionales.

La Universidad Nacional Abierta (UNA) fue creada en la década de los 70 del pasado siglo, bajo los principios orientadores de democratización, masificación, contribución al desarrollo autónomo, renovación, complementariedad, optimización y amplitud nacional.

A nivel de pregrado la UNA oferta carreras cortas -con una duración de seis semestres- $y$, carreras largas, cuya duración es de diez semestres, entre las que se encuentra la carrera objeto de este estudio: ingeniería industrial, cuyo objetivo es formar profesionales capacitados en las áreas científica, humanística y tecnológica, que sistematicen y optimicen la actividad productiva de bienes y servicios, en pro del beneficio social y económico, así como de la preservación del entorno. 
Además, esta universidad es pionera por su cobertura de acción en el ámbito nacional y en la educación a distancia, modalidad que potencia su condición de trascender sus fronteras nacionales y tener presencia en el ámbito global. De igual manera, la educación a distancia se convierte en una ventaja en el proceso de globalización, pues permite de forma rápida, segura y eficiente la expansión y profundización del conocimiento para el beneficio de la sociedad, puesto que, tal como lo refiere la Unesco (1998) "la sociedad cada vez tiende más a fundarse en el conocimiento" (s.n.)

El proceso de globalización ha abierto fronteras en diversos espacios: político, social, económico y muy particularmente en el educativo. Hoy día el profesional requiere competencias internacionales que le permitan competir y ser elegible en cualquier nivel de conocimiento que su preparación académica le haya proporcionado. Alcanzar competencias internacionales exige a su vez un diseño curricular internacionalizado, que brinde respuestas de manera eficiente y eficaz a la necesidad latente y real que hoy tienen las comunidades: comprender, vivir y trabajar en un mundo cada vez más interconectado, multicultural y tecnológicamente más avanzado.

Así, el diseño currículo se convierte en la herramienta idónea que recoge las exigencias académicas que requieren alcanzar los profesionales para adaptarse a estos nuevos entornos sociales $y$ culturales, con una mentalidad global que les permita desempeñarse, bajo preceptos éticos y morales, en cualquier organización en el ámbito internacional.
La internacionalización curricular implica, necesariamente, una flexibilidad curricular: una apertura al cambio y a la innovación que generen, tal como lo plantea Larrea y Astur (2013) "nuevos sentidos y resignificados del discurso y propician nuevas creaciones que parten de la propia cultura y política socioeducativa" (s. n).

Según la Universidad Distrital Francisco José de Caldas (2015) la internacionalización del currículo puede definirse como:

El conjunto de acciones
académicas, administrativas y
tecnológicas que una Institución
de Educación Superior
implementa transversalmente,
para la formación integral de
profesionales e investigadores
globalmente competitivos, con
identidad cultural, formados con
conocimientos y tendencias
globales para la solución de
problemas locales (s.n.).

La internacionalización curricular debe ser entendida como un proceso de transformación que involucra una apertura que irrumpe las fronteras nacionales hacia el exterior y el cual debe ser parte integral de los planes de desarrollo institucional. Alcanzarlo exige del compromiso de los actores claves del proceso de innovación y flexibilidad curricular.

En efecto, la internacionalización, como proceso de transformación, no surge espontáneamente, sino que, como proceso, debe ser planeada, integrada a la misión y a las funciones sustantivas de la universidad, a fin de que se establezcan 
estrategias de acción: académicas y administrativas para "operacionalizar el proceso y, la internacionalización de la docencia, la investigación y la acción social" (Gacel-Ávila, 2010, p. 31).

La internacionalización de la educación superior, además de constituirse en una dimensión que refuerza la preparación de los estudiantes frente a un mundo sin fronteras que se desarrolla en el escenario de la globalización, tiene como ventajas: un mayor intercambio y fortalecimiento de conocimientos; incremento de la movilidad de profesionales, docentes e investigadores para su propia actualización de contenidos a transmitir, lo cual, a su vez, mejora sus capacidades de ampliar nuevos horizontes en las diferentes áreas de las ciencias.

En tal sentido, la política de internacionalización, tal como lo plantea la Universidad del Siglo 21 (2015) se conforma en "una autovía de conocimiento en la que las instituciones educativas exportan e importan currícula académica que fortalecen los contenidos de cada una de las carreras universitarias impartidas, preparando mejores graduados en lo humano, cultural, social como en lo profesional" (s.n.).

En razón de lo expuesto, las diversas acciones de internacionalización, tales como: programas de intercambio estudiantil, convenios y cooperación para el desarrollo, pasantías a nivel de docentes, investigadores y estudiantes; cursos sobre idiomas, entre otros, implican un compromiso institucional de los diferentes autores que hacen vida universitaria, a fin de administrar el conocimiento y desarrollar currículos flexibles que propicien la homologación de planes y programas de estudios y dobles titulaciones, a manera de favorecer la movilidad académica.

En este sentido, lo antes planteado coincide con la postura de Perrotta (2014), al considerar que la internacionalización es un proceso cuyo basamento está centrado en: a) el papel que representa el conocimiento a nivel global; b) un mercado de trabajo para personas cada vez más competitivas; c) el rápido incremento proporcionado por las TIC a la interconectividad entre productores y consumidores de conocimiento.

Por consiguiente, resulta claro la importancia que reviste para una institución de educación superior desarrollar un proceso educativo partiendo de un diseño currículo que incorpore dentro de sus elementos la dimensión internacional, tal es el caso de la carrera de ingeniería industrial, implementada por la UNA.

En razón a lo expuesto, se plantea el siguiente enunciado holopráxico: ¿En qué medida se ajusta el diseño curricular actual de la carrera Ingeniería Industrial de la Universidad Nacional Abierta de Venezuela a la tendencia del tercer milenio de internacionalización del currículo para la educación universitaria?

Según la Unesco (1998), la viabilidad de la educación superior y su capacidad para transformarse y propiciar el cambio y el progreso de la sociedad ha quedado demostrada a través de los años. En la sociedad contemporánea, las transformaciones y el progreso se 
sustentan en el conocimiento, siendo uno de los medios para lograrlo, la educación superior o también denominada educación universitaria.

Así, la educación universitaria y la investigación son parte indispensable para el desarrollo social, económico, cultural y ambiental sostenible de la sociedad contemporánea, por lo que la surte de una serie de desafíos para emprender la transformación y la renovación exigida por un contexto cambiante (Unesco, 1998).

Las universidades, como instituciones cuya tarea medular es el cultivo del conocimiento, -en su más amplio sentido, están Ilamadas a establecer vínculos con el entorno con la finalidad de crear dinámicas y productivas interfaces para la promoción de programas académicos; así como, el fomento de la docencia, la investigación y la extensión, los servicios de consultorías y asesorías, los emprendimientos de educación a distancia, los grupos de investigación, entre otros. Estos vínculos impulsan los procesos de competitividad entre estas instituciones (Brunner, 2005).

La competitividad de una universidad se pone de manifiesto al contener en su plantilla de docentes reconocidos investigadores, permitir la matriculación de estudiantes de cualquier origen; difundir la calidad educativa que la caracteriza, mediante el desempeño intelectual y laboral de sus egresados, en los ámbitos que le competen; así como la optimización de los servicios y el tipo de gestión interna y externa llevada a cabo por la universidad.

En razón de lo antes planteado, es menester la integración de las funciones de docencia, investigación y extensión con un sentido amplio de responsabilidad, en aspectos económicos, sociales, culturales y de calidad educativa, no solo en el contexto local sino abarcando, también, el contexto global.

Para el logro de la competitividad las universidades hacen esfuerzos para articular sus procesos educativos con el sector empresarial, estando conscientes en que este último también ha evolucionado con los avances científicos y tecnológicos y su aplicación en los sistemas productivos (Dávila, 2008).

Es evidente, entonces, que el egresado universitario debe tener el perfil profesional para desempeñarse de manera óptima en la empresa que lo contrate, lo que supone que en el programa de estudios estén incluidos aquellos aspectos requeridos por ese sector, bien sea local, regional o global.

En ese sentido, plantea Enríquez (2005) que es indispensable que los contenidos programáticos se brinden en función de lo que el estudiante necesita aprender y no en razón de lo que el docente conoce o cree conocer. De igual manera, los docentes están obligados a "una renovación permanente de teorías, técnicas o procesos, en estrecha relación con el conocimiento que se produce dentro y fuera del contexto universitario" (p. 12).

Lo anterior alude una serie de transformaciones en el sistema de educación universitario: que los docentes y estudiantes se conviertan en permanentes aprendices; que promuevan, generen y difundan conocimientos por medio de la investigación; además, 
programas de estudios en constante revisión y adaptación, en lo que respecta a la difusión de nuevos conocimientos y las nuevas tecnologías de enseñanza y aprendizaje (Enríquez, op. cit).

En el contexto de la sociedad contemporánea, las universidades están llamadas a revisar su compromiso social, a asumir una serie de desafíos y retos orientados hacia el logro de su competitividad, el aseguramiento de la calidad educativa, el reforzamiento del contenido interdisciplinario y pluridisciplinario de los programas educativos, el mejoramiento de métodos y técnicas educativas, y el reforzamiento de la integración entre la investigación y la enseñanza en el campo científico y tecnológico (Enríquez, op.cit).

Más aún cuando, esa misma sociedad está caracterizada por una interconexión basada en una red telemática de comunicaciones que progresivamente tiene cobertura en todo el planeta. Esta red telemática es producto de las tecnologías de la información y la comunicación (TIC), las cuales han impulsado grandes transformaciones en todos los ámbitos mundiales, el ámbito universitario no escapa de esas transformaciones tecnológicas. Así, las TIC le imprimen a las universidades profundos cambios sobre sus funciones de formación, investigación y, en general, al servicio prestado a la sociedad.

En términos de la Unesco (1996), para que las universidades respondan de manera eficaz a esos desafíos y retos, que resultan en múltiples y complejos problemas, deben reorientar de manera trascendental su misión, funciones, forma de organización interna y los procesos de formación científica y profesional.

En cuanto a los procesos de formación profesional, es necesario que desde las universidades emerjan programas educativos donde el aprendizaje supere los conocimientos enciclopédicos en un campo determinado y se centren en las capacidades intelectuales y los valores universales (Enríquez, op. cit). Donde, más allá de las capacidades intelectuales, existan potencialidades técnicas que permitan al egresado universitario, ampliar su campo de dominio sobre los procesos donde se desarrolla como ente de cambio social.

En este sentido, el currículo representa un medio para lograr la articulación entre el proceso educativo universitario con las transformaciones de la sociedad. Para Soto (1977, c.p. Casanova, 2010), el currículo está relacionado, de manera íntima, con los conceptos, principios y características de los marcos teóricos de referencia de la educación. Si bien esta definición solo abarca una temática teórica referencial de la educación, manifiesta esa vinculación indispensable que existe entre el currículo y la sociedad.

Según Amadio, Opertti, y Tedesco (2014), el currículo es "el componente fundamental de todo proceso educativo, (...) donde se aborda sobre qué, para qué y cómo educar" (p. 1); esto con el fin de responder eficazmente a las expectativas y demandas de una sociedad caracterizada por las incertidumbres y grandes transformaciones. El currículo es considerado la génesis del diseño curricular de las carreras ofertadas por las 
instituciones de educación, en este caso particular las instituciones de educación superior.

Por su parte, el diseño curricular es considerado una guía para llevar un proyecto curricular a la práctica (Casarini, 1999). Es una herramienta de gestión para el proceso de enseñanza y de aprendizaje que contiene todos los elementos para hacer operativo el hecho educativo.

En el caso particular de la carrera de ingeniería industrial, esta presenta un diseño enfocado "hacia lo académico como eje de la organización curricular, las experiencias de aprendizaje del individuo y la visión tecnológica como ejes orientadores del proceso de administración del currículo" (UNA, 2005, s.n.), lo cual guarda correspondencia con el accionar de la UNA, como institución de educación universitaria en la modalidad abierta y a distancia, que atiende a la necesaria vinculación e integración universidad currículo -transformación -sociedad.

En efecto, desde las universidades deben generarse las grandes transformaciones como producto del reconocimiento universal de la cultura científico-tecnológica, para dar paso a la aceleración de los conocimientos, la eficacia de las innovaciones tecnológicas y la inclusión de las TIC en los procesos educativos. Por consiguiente, el proceso educativo universitario debe articularse con el destino de sociedad contemporánea; más aún, cuando cobra auge la globalización de estos procesos.

Para Moreira (2003), la globalización representa la superación de los límites espaciales de las fronteras locales 0 nacionales para actuar a nivel planetario, basándose en los efectos de los avances científicos y tecnológicos en materia de información y comunicación. Esto trae consigo una expresión de conocimiento a través de las redes telemáticas que, permiten la difusión de este. Además de implicar connotaciones culturales y educativas específicas (García, García y Gavari, 2012).

En las universidades, el proceso de globalización permite la interconexión con distintos ámbitos del saber, de la investigación, del conocimiento, entre otros. La globalización de la educación impulsa cambios en los estilos de enseñanza tradicionales en las universidades. Hay una transformación de los modelos educativos, de las estructuras curriculares, de las relaciones entre los sujetos con sus docentes y con sus instituciones. Transformaciones asociadas a las innovaciones científicas y sus aplicaciones tecnológicas en dichas casas de estudios.

Una sociedad centrada en el conocimiento exige a las universidades la transformación de los programas de enseñanza y los planes de estudio, con una mayor diversificación de áreas del conocimiento, el surgimiento de nuevas carreras, y una creciente interdisciplinariedad y flexibilidad en los planes de estudio.

En el plano curricular ninguna universidad se sustrae del influjo y efectos de la globalización de la educación, aunque evidentemente la naturaleza e intensidad del impacto varíen sustancialmente entre estas 
instituciones (De Zan, Paipa y Parra 2011).

De lo anterior se deriva la necesidad de planificar currículos de carreras universitarias destinados al acceso del conocimiento y a las nuevas tecnologías en los diversos sectores de la sociedad, lo cual implica integrar las en los procesos educativos y redefinir los contenidos del currículo bajo una visión local y global. En este sentido, tanto la globalización como las TIC que la impulsa, fomentan las tendencias de internacionalización del currículo.

La internacionalización se interpreta como una de las formas en que la educación universitaria reacciona a las posibilidades y desafíos de la globalización. Es a la vez un objetivo y un proceso, que permite que estas instituciones logren una mayor presencia y visibilidad internacional que genera beneficios del exterior (Siufi, 2009).

La internacionalización de la educación universitaria representa para Arum y Van de Water (1992 c.p De Zan, Paipa y Parra, 2011), todas aquellas actividades, programas y servicios que incluyen los estudios internacionales, el intercambio educativo internacional movilidad de estudiantes y profesores- y los convenios o trabajos de cooperación técnica. Estos autores sugieren tres elementos para que se internacionalice una universidad, que son los estudios universitarios, el intercambio educativo y la celebración de convenios de cooperación técnica.

En otros términos, se encuentra el planteamiento de Fernández y Ruzo (2002), quienes consideran que la internacionalización abarca aquellos aspectos de los sistemas institucionales que superan las fronteras de un país o que están bajo la influencia de las relaciones interinstitucionales entre estudiantes, profesores, gestores, sistemas, administraciones, y demás agentes en otros países.

La internacionalización del currículo implica la comprensión entre culturas distintas, el dominio de un segundo idioma, la movilidad de estudiantes, académicos y profesionales, la expansión de la oferta educativa transnacional, la asociación con instituciones nacionales, siendo un mecanismo frecuente; la doble titulación, los sistemas para evaluar y acreditar la calidad, los procedimientos de revalidas, los convenios interinstitucionales, las actividades y programas internacionales, los proyectos de cooperación para el desarrollo, la participación en redes de investigación y de docencia internacionales, administrativo, entre otros, (Altbach y Knight, 2006; Dávila, 2008; Gacel-Ávila, 2004; Moctezuma y Navarro, 2011).

En el marco de los planteamientos anteriores, se considera que en un mundo interconectado, donde los desafíos son comunes, ya no se estima, ni conviene, ni resulta suficiente que el currículo tome en cuenta solamente necesidades y prioridades nacionales. Pues, el currículo debe ser integral, flexible, donde se aborden aspectos que abstraigan necesidades locales, nacionales, regionales y globales.

Con referencia a esta investigación, el diseño curricular de la carrera ingeniería industrial fue analizado en razón del criterio internacionalización y las sinergias a considerar son: 
- Temas internacionales: referidos a que el diseño curricular contenga temas de interés internacional de manera que los estudiantes puedan determinar las tendencias del ejercicio profesional de su carrera.

- Amplitud idiomática: implica que el diseño curricular contempla la posibilidad de que los estudiantes logren un dominio de varios idiomas, tal que puedan acceder a contextos internacionales con mayor facilidad.

- Ámbito de actuación internacional: comprende aquellas actividades presentes en el diseño curricular que permitan la actuación de los estudiantes y docentes en escenarios internacionales.

- Redes internacionales: alude a la relación del diseño curricular con conexiones internacionales referidas a la divulgación del conocimiento.

- Compatibilidad con sistemas de educación superior internacional: comprende la inclusión en el diseño curricular de aspectos que permiten la compatibilidad del sistema de unidad crédito a fin de garantizar procesos administrativos de traslados entre carreras de universidades internacionales.

\section{MÉTODO}

La presente investigación se consideró de tipo analítico. En ella se analizó el contenido del diseño curricular actual de la carrera Ingeniería Industrial de la Universidad Nacional Abierta de la República Bolivariana de Venezuela, en términos de las tendencias de internacionalización. El diseño fue de tipo documental, transeccional contemporánea.
El criterio de análisis fue la internacionalización del currículo. La técnica aplicada fue la revisión documental y se utilizó como instrumento de recolección de datos una matriz de análisis elaborada en función del criterio de internacionalización. La unidad de estudio estuvo conformada por el documento denominado: Ajuste curricular de la carrera ingeniería industrial vigente (2005-2017). Aprobado según Gaceta Universitaria, Universidad Nacional Abierta, Año 2005, 11/07/2005, Número 041 Extraordinario; así como las asignaturas contenidas en el plan del estudio referidas a las áreas de conocimiento: básicas, básicas de ingeniería industrial, formación profesional y práctica profesional.

\section{RESULTADOS}

\section{Criterio de análisis: Internacionalización del diseño curricular}

Las sinergias analizadas, correspondientes a la internacionalización del currículo de la referida carrera universitaria, fueron: temas internacionales, amplitud idiomática, ámbito de actuación internacional, redes internacionales y compatibilidad con sistemas de educación superior internacional.

Temas internacionales. Con relación a esta sinergia se constató que en el objetivo de la carrera no se plantea el dominio de temas internacionales, puesto que la Ingeniería Industrial declara como objetivo:

a) Promover en el egresado de la UNA la comprensión de la realidad social, 
política, económica, científica, tecnológica y cultural de Venezuela; b) Promover el aprendizaje de sistemas, métodos y técnicas para la comprensión y solución de problemas; c) Vincular, por vía de integración progresiva, la problemática objeto de estudio del componente profesional con el contexto del egresado.

Por otra parte, según lo plasmado en el documento Ajuste curricular la carrera Ingeniería Industrial tiene como función:

la integración y optimización de los recursos: humanos, materiales, económicos, de información y energía en los sistemas industriales y de servicios; así como incrementar la productividad, calidad, servicio y rentabilidad de los sistemas de actividad humana, para lograr una mayor competitividad, un mejor nivel de vida y bienestar social de los integrantes de los sistemas y de la población en general (p. 1)

Puede notarse que tanto el objetivo como la función de dicha carrera están totalmente desvinculados. Además, en razón de lo declarado en la función se deduce que: el objetivo de la carrera, el perfil del egresado, la estructura curricular, la administración y la investigación, como elementos importantes del Ajuste curricular de la carrera, no denotan aspectos que los vinculen con el abordaje y dominio de problemáticas internacionales, que incluyan culturas de otros países desde la multidisciplinariedad; e incluir, de ser necesario, publicaciones extranjeras en revistas, sitios web, entre otros, a fin de poder considerar el programa de la referida carrera en concordancia con la tendencia de internacionalización.

Asimismo, al analizar el plan de estudio en relación a la sinergia temas internacionales, se verificó que en su mayoría las estrategias instruccionales no están especificadas o limitadas a contexto geográfico alguno. En estas estrategias tampoco se incluye la realización de estudios comparativos y multidisciplinarios ni se propicia la participación de los estudiantes con ensayos, artículos, informes, entre otros, en publicaciones extranjeras de revistas, sitios web, entre otros.

Las bibliografías utilizadas en los planes de curso son, en su mayoría, textos de mercado editados en otros países. Lo que a juicio de la investigadora no representa indicios de internacionalización.

Igualmente, se constató en los planes de curso que se cuenta con contenidos sistemáticos universales para el diseño e implementación de los planes y programas de estudios. Esto representa una proyección o explicitación del proceso formativo conducente a consolidar el perfil de egreso, describiendo los contenidos con base a problemáticas, en primer momento nacional para luego, en algunos casos, trabajar con problemáticas globales; además, se consideran actividades teóricas y prácticas de manera consistente e integrada.

Aun cuando para el logro del perfil de egreso, los planes de curso cuentan, cuando sea necesario, con alianzas efectivas con empleadores para desarrollar prácticas profesionales de calidad, 
durante el desarrollo del programa, de modo que los estudiantes desarrollen conocimientos, habilidades y la disposición necesaria para ejercer eficazmente su futura actividad laboral, esta se limita al territorio venezolano, no a instancias internacionales.

Al respecto, Glazman e Ibarrola (1978) plantean que "el plan de estudios es la síntesis instrumental mediante la cual se seleccionan, organizan y ordenan, para fines de enseñanza, todos los aspectos de una profesión que se considera social y culturalmente valiosa, profesionalmente eficiente" (p. 13).

En efecto, se entiende que los planes de curso deben responder a la dinámica social venezolana en consonancia a dar respuestas eficaces a las problemáticas existentes en el contexto laboral; sin embargo, no se promueve escenarios y oportunidades para que esos procesos y experiencias sean sistematizados en artículos científicos, en revistas arbitradas para la difusión del conocimiento y así adentrase en la sociedad de la información.

Al mismo tiempo, los planes de curso incluyen experiencias de aprendizaje que apuntan al desarrollo de competencias transversales, tales como: comunicación oral y escrita, pensamiento crítico, solución de problemas, desarrollo de relaciones interpersonales, autoaprendizaje e iniciativa personal, trabajo en equipo y uso de tecnologías de información, solo orientado a la ejecución en el contexto venezolano.

Por lo cual, se concluye que la carrera no incluye temas internacionales. Es importante señalar que la internacionalización del currículo no desestima el desarrollo local, por el contrario, según establece Knight (2012) la internacionalización es el "proceso de integrar una dimensión internacional, intercultural o global dentro del propósito, funciones u oferta de la educación superior" (p. 14). Es decir, este principio no desestima a los actores, acciones y resultados que varían significativamente en virtud de sus contextos particulares que recogen características propias de cada espacio territorial en el cual se desarrolle el proceso formativo.

Cabe destacar que, a pesar de no aparecer la pertinencia social en la estructura del diseño de la carrera, los principios del currículo de la UNA establecen la pertinencia social dirigida hacia la búsqueda de un profesional con una visión cósmica, holística, que considere la calidad de vida y preservación del medio ambiente, para la actual sociedad global. Este elemento, la pertinencia social, no se aprecia en el objetivo de la carrera, ni en el perfil del egresado y tampoco forma parte de su estructura curricular.

Amplitud idiomática. Con respecto esta sinergia, es importante destacar que en el perfil de egreso se expresa la obligatoriedad de aprobar la asignatura inglés (Cód. 108); sin embargo, el alcance del curso se limita a aplicar técnicas para la deducción del significado de palabras desconocidas y para la comprensión de textos técnicos y científicos en inglés; por tanto, no se exige un nivel conversacional o avanzado del idioma extranjero.

Por otra parte, se ofrece el curso lengua y comunicación, cuyo objetivo se 
centra en distinguir de manera precisa las acciones comunicativas, tanto personales como las relacionadas con el paradigma teleinformática a partir de las manifestaciones concretas de dichas acciones; por tanto, aborda características básicas del proceso de comunicación y del lenguaje, pero no ofrece la posibilidad de enseñar español a extranjeros.

De lo anterior se concluye que el diseño de la carrera no incluye el dominio de otros idiomas. En ese sentido, el PhD. Olman Segura, ex rector de la Universidad Nacional de Costa Rica, (c. p. España 2010, 67), afirma que:

\section{La inclusión de cursos de inglés y de tecnologías de informática en el currículo de las diferentes carreras es parte de esta internacionalización... tomando en cuenta que en el mundo globalizado actual el manejo de este idioma, de uso universal, y el adecuado uso de las tecnologías de la información y la comunicación son herramientas esenciales que requieren todos los y los profesionales para vincularse exitosamente al mercado laboral.}

Considera, además, España (2010) que: "hoy en día es evidente que el manejo de una sola lengua no es suficiente para responder a las demandas que impone un mundo interconectado, el cual ve impactadas las relaciones de trabajo, estudio y convivencia por el idioma" (p. 65). Lo que hace necesario considerar en la carrera de ingeniería industrial que se desarrolla actualmente la formación en lenguas extranjeras y nativas, pues es un elemento ausente, desde la mirada de la internacionalización del currículo.

Ámbito de actuación internacional. En los hallazgos encontrados en la sinergia antes referida, se constató que el diseño curricular de la carrera ingeniería industrial de la UNA no se corresponde con un ámbito de actuación internacional. En el mismo no se incluye el reconocimiento internacional de los egresados, ni la movilidad académica internacional que promueva el intercambio de docentes y estudiantes. Además, no se contemplan prácticas en el extranjero, ni programas y cursos internacionales a lo largo de los semestres.

Es importante señalar que de acuerdo a Rama (2012), la internacionalización de la educación superior "se expresa a través la movilidad de estudiantes y docentes, que es la forma tradicional asumida como cooperación; y por el traslado e instalación local de instituciones extranjeras y la educación transfronteriza a través de la enseñanza virtual" (p. 3).

La movilidad académica de estudiantes, profesores e investigadores, según Madarro (2011) "constituye una oportunidad para las personas en lo que se refiere a la formación, al desarrollo de capacidades en la actual sociedad del conocimiento y a la innovación tecnológica" (p. 71). Por lo que, los futuros profesionales e investigadores, deben tener la oportunidad de realizar un periodo de estudios en una universidad extranjera, reconocidos estos estudios, por las instituciones de origen involucradas, como parte de su formación académica. 
Dentro de las actividades que se consideran como posibilidades de movilidad de estudiantes, investigadores, docentes y personal administrativo, destacan, de acuerdo a la Universidad Pedagógica de Colombia (2016), las siguientes:

(a) Intercambio institucional 0 académico recíproco, (c) semestre académico en el exterior, (d) Transferencia académica, (e) Estudios de postgrado, (f) Experiencia inter-cultural, (g) Prácticas empresariales,

Perfeccionamiento de una segunda lengua, (i) Pasantía internacional, (j) Conferencias y eventos especiales. (s.n.)

Por lo cual, los diseños curriculares deben proveer dentro de su génesis la posibilidad, al estudiante, de realizar alguna de las actividades descritas anteriormente, y que en el caso específico de la carrera que se está analizando, no se encuentran hallazgos dentro de los documentos oficiales que demuestren su consideración.

Es importante señalar que la vinculación con el medio laboral es un componente esencial del quehacer de la carrera o programa que orienta y fortalece el perfil de egreso y el plan de estudios. Existe una interacción sistemática, significativa y de mutuo beneficio con agentes públicos, privados y sociales relevantes, de carácter horizontal y bidireccional.

En aras de lo anterior, se enlazan políticas y mecanismos de evaluación periódica de impacto de las actividades de vinculación con el medio promoviendo actividades académicas con vías a propiciar los intercambios amenos entre la universidad y empresa, para fortalecer la formación. Sin embargo, no existe la apertura de que estos intercambios transciendan a las dinámicas globales, puesto que los convenios y recursos son limitados.

La Unesco (2010) expone que los planes de estudio de la carrera de ingeniería deben orientarse a: (a) difundir información sobre la manera como han manejado las problemáticas en diversos países; (b) incentivar a las instituciones a la revisión de sus planes de estudios y contextualizarlos al mundo globalizado. Desde esta mirada, los planes de estudios de ingeniería deben mostrar un mapa de vinculaciones globales, en concordancia a mostrar la coincidencia fundamental en los tipos de problemas que surgen en los procesos industriales y en los métodos empleados para resolverlos.

La información sobre los procedimientos que han dado resultados satisfactorios en otros países puede servir para estimular y alentar a las autoridades locales a adoptar iniciativas para el intercambio permanente de experiencias laborales, a través de cursos, programas de intercambio estudiantes, plataformas virtuales para publicaciones.

Redes internacionales. En esta sinergia se hizo evidente que en el diseño curricular de la carrera ingeniería industrial de la UNA, hay una total ausencia de vínculos con estudiantes extranjeros; de actividades académicas en el exterior o asistencia a eventos internacionales, como tampoco suscripción 
de convenios internacionales; por lo cual, el ámbito de actuación del docente y el estudiante se reduce al nacional.

Es importante señalar que no todos los países tienen a su disposición los mismos medios para emprender y financiar actividades académicas en el extranjero, por ello, la educación incumbe sólo al personal docente y consiste en exponer datos, recitar lecciones y hacer exámenes.

En este sentido, la Conferencia Mundial de Educación Superior realizada en París, en el 2009 (CMES-2009), destacó la importancia fundamental de la cooperación internacional solidaria como vía para mejorar la calidad de la educación superior, así como su contribución a la reducción de la brecha en materia de desarrollo, mediante el aumento de la transferencia de conocimientos.

La cooperación académica internacional, como ámbito específico de la cooperación internacional, ha sido definida por Sebastián (2004), como:

El conjunto de actividades realizadas entre instituciones universitarias que, a través de múltiples modalidades, implica una asociación y colaboración en temas de política y gestión institucional; la formación, la investigación, la extensión y la vinculación para el mutuo fortalecimiento y la proyección institucional; la mejora de la calidad de la docencia; el aumento y la transferencia del conocimiento científico tecnológico; y la contribución a la cooperación para el desarrollo (p. 56).

Las actividades antes señaladas son de gran importancia para el futuro profesional, puesto que le permite ampliar sus horizontes, sobre todo en una sociedad globalizada y en la que las tecnologías de la información y las comunicaciones han reducido las fronteras, particularmente en espacios virtuales, que brindan nuevas herramientas para aprender.

Resulta claro que en una universidad como la UNA, caracterizada por su modalidad a distancia, es de suma importancia incrementar el conocimiento y el desarrollo, así como las posibilidades de acuerdos para el establecimiento de programas conjuntos de intercambio que incrementen el sentimiento de pertenencia a la profesionalidad y enriquezcan la formación de los estudiantes, docentes e investigadores en el marco de la integración global.

Compatibilidad con sistemas de educación superior internacional. Las universidades tienen asociaciones institucionales de carácter bilateral, multilateral para la interacción, colaboración con base a la titulación y homologación de las asignaturas. En América Latina y el Caribe existen numerosas asociaciones, redes regionales, subregionales, institucionales de Educación Superior y de universidades.

Estas organizaciones desarrollan diferentes tipos de actividades de cooperación interuniversitarias que se traducen en iniciativas multilaterales, de acuerdos intergubernamentales bilaterales, 
convenios interinstitucionales, entre otras, todo con el fin de validar los programas de estudios, las mallas curriculares y certificaciones de participaciones del estudiante en las regiones en la cual se formó. Es de aclarar que las asignaturas no requieren mostrar en su totalidad temáticas de interés internacional puesto que abordan las ciencias universales, las cuales son tituladas y certificadas en el extranjero.

El Diseño Curricular de ingeniería industrial de la UNA (2005-2017) expresa "el currículo deberá vincularse con sentido de pertenencia social y prospectiva con su entorno: local; regional; nacional; latinoamericano integrado" (p. 18), sin embargo al analizar los planes y programas de la citada carrera se confirma que en los mismos se da gran relevancia al desarrollo del sentido de pertinencia hacia lo local y nacional, lo cual pone en evidencia que el diseño curricular de la carrera de ingeniería industrial no guarda compatibilidad con los sistemas de educación superior internacionales.

\section{CONCLUSIONES}

Al analizar el documento Ajuste curricular de la carrera ingeniería industrial (2005-2017) con relación a la sinergia denominada temas internacionales, se determinó que: el objetivo y función de la carrera se encuentran desvinculados. Asimismo, el análisis del plan de estudio demostró que en su mayoría las estrategias instruccionales no están especificadas o limitadas a contexto geográfico alguno. Las bibliografías utilizadas en los planes de curso son, en su mayoría, textos de mercado editados en otros países, lo cual no representa indicios de internacionalización.

De igual manera, se evidenció que los planes o programas de curso abordan problemáticas nacionales y solo en algunos casos situaciones globales. El perfil del egresado está limitado al entorno nacional. En razón de lo expuesto se pudo evidenciar que el objetivo de la carrera, el perfil del egresado, la estructura curricular, la administración y la investigación, elementos medulares del ajuste curricular, no incluyen temas internacionales.

Con respecto a la sinergia amplitud idiomática, es importante destacar que, en el perfil de egreso se expresa la obligatoriedad de aprobar la asignatura inglés (Cód. 108); sin embargo, el alcance del curso se limita a aplicar técnicas para la deducción del significado de palabras desconocidas y para la comprensión de textos técnicos y científicos en inglés; por tanto, no se exige un nivel conversacional o avanzado del idioma extranjero.

Por otra parte, se ofrece el curso Lengua y comunicación, cuyo objetivo se centra en distinguir de manera precisa las acciones comunicativas, tanto personales como las relacionadas con el paradigma teleinformática a partir de las manifestaciones concretas de dichas acciones; por tanto, aborda características básicas del proceso de comunicación y del lenguaje, pero no ofrece la posibilidad de enseñar español a extranjeros.

Con relación a la sinergia ámbito de actuación internacional, los hallazgos encontrados permitieron constatar que el diseño curricular de la carrera ingeniería industrial de la UNA no se corresponde con 
un ámbito de actuación internacional. En el mismo no se incluye el reconocimiento internacional de los egresados, ni la movilidad académica internacional que promueva el intercambio de docentes y estudiantes. Además, no se contemplan prácticas en el extranjero, ni programas y cursos internacionales a lo largo de los semestres.

El análisis del diseño curricular de la carrera ingeniería industrial de la UNA con respecto a la sinergia redes internacionales, evidenció que hay una total ausencia de vínculos con estudiantes extranjeros; de actividades académicas en el exterior 0 asistencia a eventos internacionales, como tampoco suscripción de convenios internacionales; por lo cual, el ámbito de actuación del docente y el estudiante se reduce al nacional, en razón de lo planteado se demuestra que el diseño curricular de la carrera no evidencia aspectos que lo vinculen con redes internacionales.

Finalmente, a la luz del análisis de los planes y programas de la citada carrera, se confirmó que en los mismos se da gran relevancia al desarrollo del sentido de pertinencia hacia lo local y nacional, lo cual pone en evidencia que el diseño curricular de la carrera de ingeniería industrial no guarda compatibilidad con los sistemas de educación superior internacionales.

\section{REFERENCIAS}

Altbach, P. y J. Knight. (2006). Visión panorámica de la internacionalización en la educación superior: motivaciones y realidades. Perfiles educativos
28.112: 13-39. México: Universidad Nacional Autónoma de México, UNAM Altbach, P. (2008). Funciones compleja de las universidades en la era de la globalización. La educación superior en el mundo 2008: La financiación de las universidades. Barcelona: Universidad Politécnica de Cataluña, UPC

Amadio, M., Opertti, R., y Tedesco, J. (2014). Un currículo para el siglo XXI: Desafíos, tensiones y cuestiones abiertas. Investigación y Prospectiva en Educación. París: UNESCO

Brunner, J. (2005). Tendencias recientes de la educación superior a nivel internacional: marco para la discusión sobre procesos de aseguramiento de la calidad. Documento de trabajo. Viña del Mar: Universidad Adolfo Ibáñez

Casanova, I. (2010). Estado del Arte del enfoque por Competencias en el contexto latinoamericano. Universidad del Zulia. Venezuela. Recuperado de https://www.researchgate.net/publicatio n/314090129_Estado_del_Arte_del_E nfoque_por_Competencias_en_el_conte xto latinoamericano

Casarini, M. (1999). Teoría y diseño curricular. México: Universidad Virtual, Itesm

Dávila, M. (2008). Tendencias internacionales de la Educación Superior. Documento de Trabajo $\mathrm{N}^{\circ}$ 219. Argentina: Universidad de Belgrano

De Zan, A; Paipa, L. y Parra, C. (2011). Las competencias: base para la internacionalización de la educación superior. Revista Educación en Ingeniería 6.11. 44-54. Colombia: Asociación Colombiana de Facultades de Ingeniería, Acofi

Enríquez, J. (2005). Educación superior: tendencias y desafíos. Educación médica 9.1: 06-10. Barcelona: Fundación Educación Médica. Disponible en: 
http://scielo.isciii.es/pdf/edu/v9n1/colab oracion2.pdf

España, C. (2010). El idioma inglés en el currículo universitario: importancia, retos y alcances. Revista Electrónica Educare, vol. XIV, núm. 2, juliodiciembre, 2010, pp. 63-69. Costa Rica: Universidad Nacional Heredia

Fernández, S. y Ruzo, E. (2002). Los procesos de internacionalización y globalización en la educación superior: Un análisis de los países Ocde. Revista de Educación. Madrid: Ministerio de Educación, Cultura y Deporte.

Gacel-Ávila, J. (2010). La internacionalización de las universidades mexicanas. Políticas y estrategias institucionales. México: ANUIES. Recuperado de

https://www.ses.unam.mx/curso2009/mat eriales/m6/LecturaComplementaria/M6 Complemetaria Gacel.pdf

Gacel-Ávila, J. (2004). La internacionalización de la Educación superior en América Latina: el caso de México. Cuaderno de Investigación en la Educación, 20. Puerto Rico: Universidad de Puerto Rico Recinto de Río Piedras, UPRRRP

García, J; García, M y Gavari, E. (2012). La educación comparada en tiempos de globalización. Madrid: Universidad Nacional de Educación a Distancia

Glazman R. y De Ibarrolla, M. (1978). Diseño de planes de estudio. México. CISE-UNAM

Henao, K. y Samoilovich, D. (2010). La Internacionalización del Currículo: ¿alternativa de la movilidad académica internacional? lesalc informa de educación superior. UNESCO, lesalc

Knight, J. (2012). Internacionalización de la Educación Superior: Nuevos desarrollos y Consecuencias No Intencionadas. lesalc informa de educación superior. UNESCO, lesalc
Larrea M. y Astur, A. (2013). Política Internacional de la Educación Superior. Acciones del Programa de Internacionalización de la Educación Superior y Cooperación Internacional 2003. 2012. En: Rinesi, Eduardo (coord.) Ahora es cuándo. Internacionalización e integración regional universitaria en América Latina. Los Polvorines: Universidad Nacional de General Sarmiento. Argentina. Recuperado de http://docplayer.es/60928161-Lainternacionalizacion-e-innovacioncurricular-de-la-educacion-universitariatendencias-perspectivas-y-desafios.html

Madarro, A. (2011). Redes de movilidad académica para la cooperación e integración regional en Iberoamérica. Revista Iberoamericana de educación. N. ${ }^{\circ} 57$

Moctezuma, P. y Navarro, A. (2011). Internacionalización de la educación superior: aprendizaje institucional en Baja California. Revista de la educación superior, 40(159), 47-66. México: Anuies

Moreira, M. (2003). Problemas y retos educativos ante las tecnologías digitales en la sociedad de la información. Quaderns digital

Perrotta, D. (2014). El Regionalismo de la Educación Superior en el MERCOSUR como vector de internacionalización: un acercamiento desde la política regional de acreditación. Recuperado de https://www.researchgate.net/publicatio n/306183450_El_regionalismo_de_la educacion_superiōren_el_MERCŌ̄JUR _como_vector_de_internacionalizacion un_acercamiento_desde_la_politica_reg ional_de_acreditacion_Regionalism_of_ higher_education_as_a_vector_of_in

Rama, C. (2012). La internacionalización ante el bien público en América Latina: entre las ideas y las realidades. En 
Mora, J. y Rama, C. (2011) Nuevos rumbos de la educación superior en América Latina: bien público, autonomía e internacionalización. Cuaderno de Ciencias Sociales No 157 , San José: FLACSO

Sebastián, J. (2004). Cooperación e Internacionalización de las Universidades. Argentina: Editorial Biblos

Siufi, G. (2009). Cooperación internacional e internacionalización de la Educación Superior. Educación Superior y Sociedad 14.1: 119-146

UNESCO. (2010). La escasez de ingenieros supone un peligro para el desarrollo, según el primer informe mundial de la UNESCO sobre la ingeniería. UNESCO, lesalc

UNESCO (1998). Declaración mundial sobre la educación superior en el siglo XXI: visión y acción. Recuperado de http://www.iesalc.unesco.org/ess/index. php/ess3/article/view/171

UNESCO (1996). Conferencia Regional sobre Políticas y Estrategias para la Transformación de la Educación Superior en América Latina y el Caribe: ideas, problemas y propuestas. Recuperado de https://unesdoc.unesco.org/ark:/48223/ pf0000219410
Universidad Distrital Francisco José de Caldas. Documento Institucional. (2015, s/n). Bogotá. Colombia

Universidad Nacional Abierta. (2005). Ajuste del diseño curricular de la carrera Ingeniería Industrial. Caracas: Universidad Nacional Abierta, UNA

Universidad Nacional Abierta. (2005). Gaceta universitaria - Universidad Nacional Abierta - Número 021 extraordinario: Ajuste Curricular de la carrera Ingeniería Industrial. Caracas: Universidad Nacional Abierta, UNA

Universidad Pedagógica de Colombia. (2016). Relaciones internacionales y cooperación interinstitucional. Recuperado de http://www.uptc.edu.co/unidad_relacion es_internacionales/movilidad/

Universidad Siglo 21 (2015). La internacionalización de la Educación: ventajas de importar y exportar estructuras curriculares. Argentina. Recuperado de https://identidad.21.edu.ar/lainternacionalizacion-de-la-educacionventajas-de-importar-y-exportarestructuras-curriculares/ 